\title{
SMART HOME AUTOMATION AND SECURITY SYSTEM DESIGN BASED ON IOT APPLICATIONS
}

\author{
Shameem Ahmad ${ }^{1}$, Arghya Saha ${ }^{2}$, Leong Wen Chek ${ }^{1}$, Saad Mekhilef ${ }^{1}$, Tariful \\ Azam $^{3}$, Mahrous Ahmed ${ }^{4,5}$, Mohamed Orabi ${ }^{5}$, Sherif Ghoneim ${ }^{4,6}$, Mosleh Alharthi ${ }^{4}$, \\ Farhan Salem ${ }^{4}$, and Basem Alamri ${ }^{4}$ \\ ${ }^{1}$ Power Electronics and Renewable Energy Research Laboratory (PEARL), Department of Electrical \\ Engineering, University of Malaya, Kuala Lumpur, Malaysia, \\ e-mail: shameem.ahmad@hotmail.com, leong@um.edu.my, saad@um.edu.my \\ ${ }^{2}$ Renewable Energy and Technology, Institute of Energy, University of Dhaka, Dhaka, Bangladesh, \\ e-mail: arghyasaha2013@gmail.com \\ ${ }^{3}$ Department of Electrical and Electronic Engineering, American International University-Bangladesh \\ Dhaka, Bangladesh, e-mail: azamtariful0@gmail.com \\ ${ }^{4}$ Electrical Engineering Department, College of Engineering, Taif University, Saudi Arabia, \\ e-mail: meahmed7@gmail.com,m.harthi@tu.edu.sa, salem_farh@yahoo.com,b.alamri@tu.edu.sa \\ ${ }^{5}$ APEARC, Faculty of Engineering, Aswan University, Aswan, Egypt, e-mail: orabi@ieee.org \\ ${ }^{6}$ Electrical Department, Faculty of Industrial Education, Suez University, Suez, Egypt, \\ e-mail: s.ghoneim@tu.edu.sa
}

Received Date: March 11, 2019; Revised Date: October 19, 2019; Acceptance Date: November 20, 2019

\begin{abstract}
Conventional Home Automation Systems lack many of the essential properties of home automation system, which are now easily accessible with modern technologies like IoT where every device is connected and can be controlled through internet. The proposed system presents a standard home automation system with all requirements an automation system has like - scalable, configurable, DIY and serves the most important function which is plug and play. Firstly, this paper discuss the merits and demerits of mainstream works on home automation system. Next, key enabling technologies - Cloud (Pubnub), Android Applications, WiFi Module (ESP 8266), Raspberry Pi have been discussed. User interfaces for web, $\mathrm{pc}$ and mobile devices are designed for this system. Also, algorithm has been constructed for connecting Wi-Fi/Bluetooth module with the system to control wirelessly. Smart home security system has been added by using PIR motion Sensor. Lastly, data have been measured from the different sensors and power consumption of the devices were calculated and showed on a graph. . Then, electricity bill consumption has been calculated and sent by sms to the user. Global and local accessing from web interface and Android Application to control devices and configurable feature (change as per require) makes the system a standard home automation framework.
\end{abstract}

Keywords: Android, Cloud, Home automation system, Raspberry Pi, WiFi Module ESP8266

\section{Introduction}

The smart home concept refers to the ability of controlling all home appliances smartly (less human interaction). This technology was used to control HVAC (Heating, Ventilation and AirConditioning) as well as fire safety and security, control by a central computer [1]. Recent evaluation of IoT technology has made possible this technology to provide various features for security, surveillance, lighting, energy management, access control, entertainment-appliances, communication, interfaces and software [2-4]. Although, it is a decade old concept, unfulfilled technical requirements including plug and play, scalability, flexibility, reliability, safety, standard wireless home automation protocol, diagnostics services to track potential problems which makes it an interesting research area [5].

Recent researchers represent different approaches focusing on performance, security and energy awareness issues to remove the barriers. The Raspberry Pi (a single board computer) integrating with Arduino (open source platform which provides microcontroller 
boards and IDE) consider as a promising solution to develop a prototype for home automation [6-11]. In [12], distributed discovery protocol has been implemented using a plug and play enabled architecture. However, his study did not offer control and network protocols. Milton and Khan [13] proposed a remote exploration and control system using web application, web server, database, GSM and Android mobile phone. Connecting the phone with electrical devices to control devices makes the system costly and requires expert installation. In [14], a Bluetooth base home automation system is presented. The system uses primary controller to connect with sub controllers physically installed on individual devices. Connecting single Bluetooth controller with numerous devices causes a delay and short range of Bluetooth networks limit. Al-ali and Al-rousan [15], developed a JAVA based automation system integrated in personal computer based web server acting as central controller. In [4], JAVA on Raspberry Pi, using Pi as sensor web node has been built for home automation system. Pi4J library is used to access Raspberry Pi by JAVA. JAVA provides the system with built-in security, but limits the flexibility of the system [17]. Since JAVA requires JVM to run the java applications, every time the system reboots, it requires to start the program manually. Though this issue can be solved by configuring boot menu of the $\mathrm{Pi}$, it requires expert technician.

Although, many companies (insteon, iSmartAlarm, Amazon, Philips) provides home automation but, most of them provide services as discrete kits (lighting kits, security kits, weather kits) which makes the system costly. In addition, they are still not adaptable in every environment specifically old infrastructures. A comparison among various technologies in major properties of home automation - networking technologies privacy issues, and their degree of desirability has been conducted in [5]. To Interface the home automation system with networking technologies, web application and mobile device application which are rated as the most degree of desirability are both used to control the appliances in local and global network $[18,20]$. Another feature is that the proposed system achieved is the providing of the encrypted text to store user data. The PubNub [16] is used as data streaming technology to provide built in Enterprise-Grade Security with other features of modern home automation systems. From above discussion, it is understood that proposed system meets all the requirements which makes it different and ideal from other home automation systems.

At first, the paper discussed about the literature review. Enabling technologies such as cloud, android application Wi-Fi module are presented in Enabling Technologies. Next section is about system architecture and last section contains the results and discussion part of the paper. In the conclusion, the major findings of the work has been presented and what can be done in the future are also described.

\section{Enabling Technologies}

IoT provides networking to connect people, applications, and data through the Internet to enable remote control, management, and interactive integrated services. That's why IoT is so important in modern home automation as it provides all the features of IoT [21-23]. Figure 1 shows proposed smart home system. To make the system cost effective and attractive to the potential users, web based and mobile application based controllers are used for both global and local users. Free cloud system is used in the system to stream the data between internet and central controller-Raspberry Pi.

\section{Cloud}

Communicating from local network is easier than communicating from internet applications. Cloud services are only cost effective options for this kind of services. In proposed system, PubNub is used as cloud system since it considers as a platform specific SDK, built-in security (multiple data encryption standards like AES and TLS/SSL) and free service. JavaScript SDK is used for web application while Python SDK is used for server side applications. 


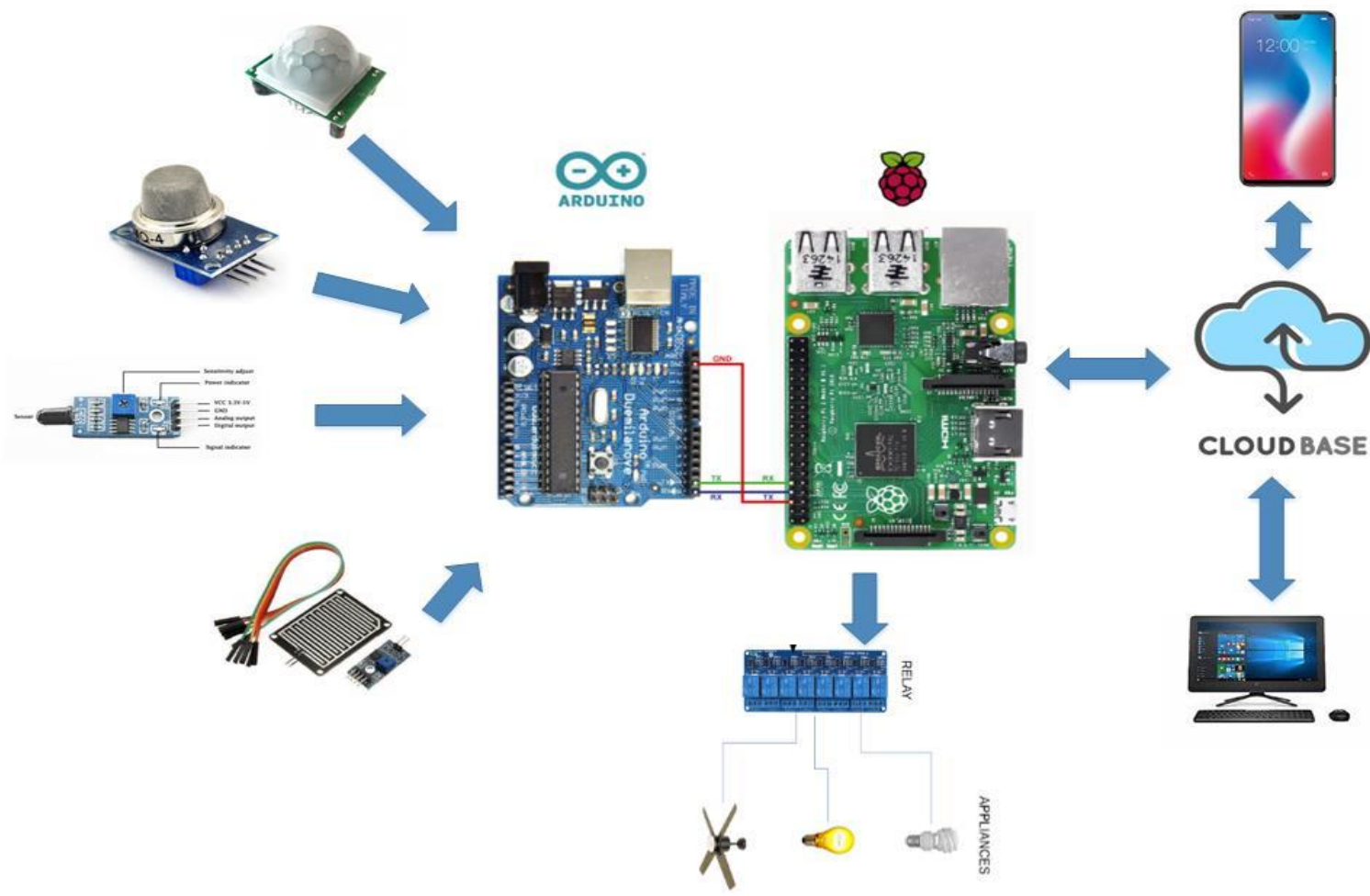

Figure 1. Proposed smart home system

\section{Android Applications}

Open operating system Android application based controller is used as Android mobile platform (smart phone, tablet) in the proposed system. While, open source language JAVA is used to develop the Android application.

\section{WiFi Module}

Wireless M2M (machine to machine) networking is the only solution for any wireless and plug and play automation systems. Bluetooth technology has some limitations as mentioned in above section. Therefore, WiFi Module- ESP8266 is used for wireless networking between node devices and central controller. IEEE $802.11 \mathrm{~b} / \mathrm{g} / \mathrm{n}$ protocol for wireless local area network (WLAN), integrated minimum 512K flash disk with additional Bluetooth coexistence interfaces and pre-programmed firmware give the designer full freedom to use it as per requirement. IoT provides low cost, low power compared to other networking shields. This module is also used for other custom WiFi modules such as - SparkFun Blynk BoardESP8266, XBee Wi-Fi OEM module. ESP8266 v.4 is used in the proposed system.

\section{PIR Motion Sensor}

Human Beings emits thermal energy of wavelength around 9-10 micro-meter every day. Pyro electric or Passive Infrared Sensor (PIR) [24], [25] is an electronic device which is designed to detect this IR wavelength when a human being is in its proximity. To have a wide range for detection a simple lens is used. Sensors may also be calibrated in such a way so as to ignore domestic pets by setting a higher sensitivity threshold, or by ensuring that the floor of the room remains out of focus Figure 2 shows the working principle of PIR motion sensor. 

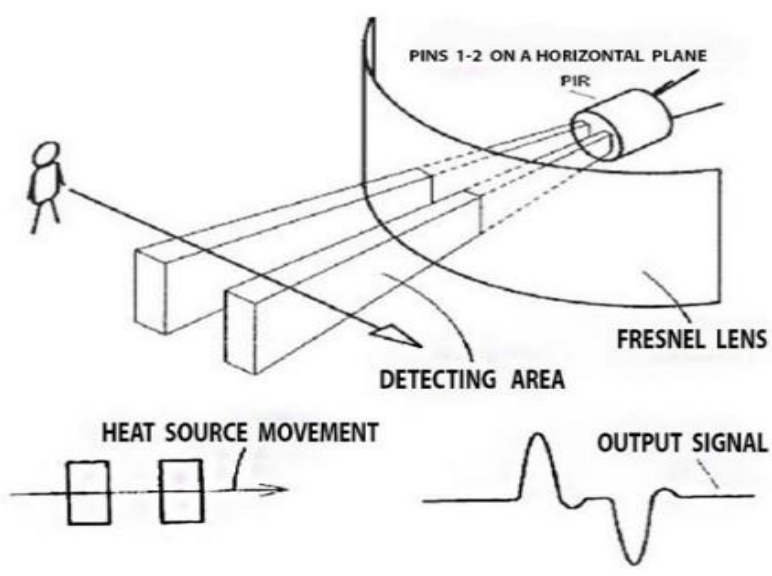

Figure 2. Working principle of PIR sensor

\section{GAS Leaking Warning System}

The proposed model can detect if there is fire or not by a smoke sensor which is shown in Figure 3. It is an electronic device which measures the smoke density and a signal is sent to micro-controller. Then according to the micro-controller set point, one can modify the output like enabling a buzzer so that there is an indication of gas leakage in one's home. Figure 4 shows the connection pin diagram by which one can set it with Arduino or any microcontroller.

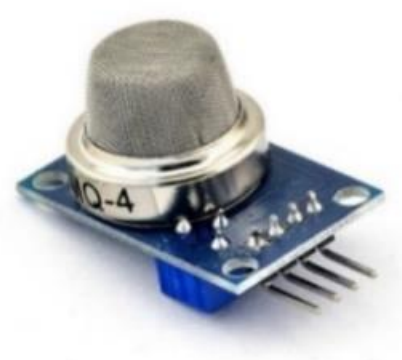

Figure 3. Arduino compatible smoke sensor
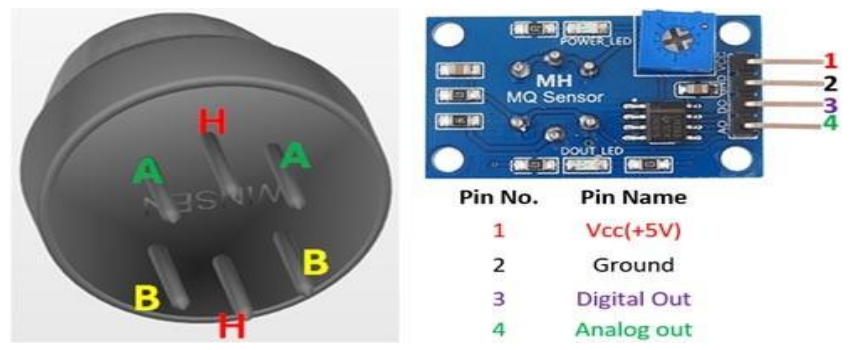

Figure 4. Connection diagram of smoke sensor with Arduino

\section{Fire Alarm System}

Infrared based Photo transistor with a flame sensor LM393 has been used to detect any kind of flame in home [26]. When any flame is detected then the digital output of the sensor is sent to microcontroller and necessary actions are taken to notify the user. 


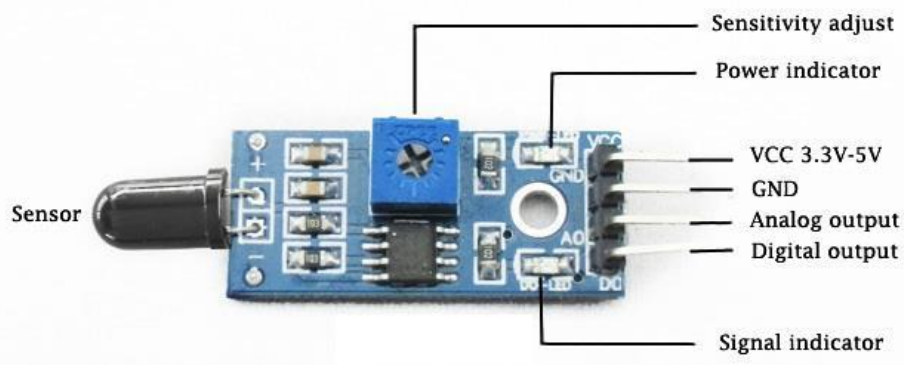

Figure 5. LM393 Flame Sensor

\section{Rain Sensing}

M009 rain sensor has been used to identify rain. When the rain drops on its surface, there is a full path of conducing current. By this the module receives information about rain and a signal is sent to the micro controller to and user is notified.

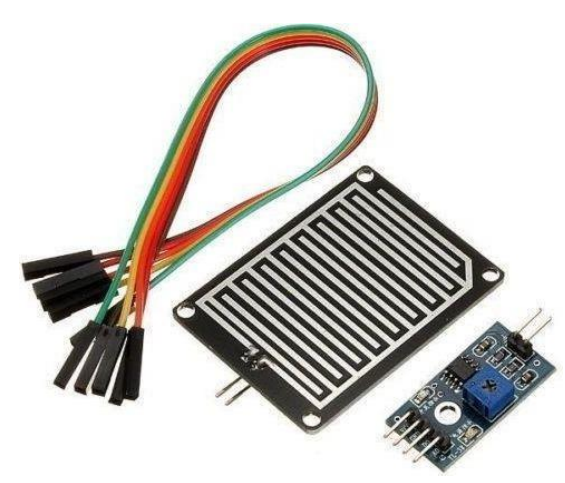

Figure 6. Rain Drop Sensor

\section{Raspberry Pi}

Raspberry Pi is a single board computer, a mainstream system subject to widespread availability and low cost than other single board computers (BeagleBone Black, Cubieboard, BananaPi). With ARM Cortex processor \& 512 MB RAM, a Raspberry Pi 2 is more adequate board for home automation systems. The amounts of work done using Raspberry Pi platform in multiple areas and their outcomes proves it as a reliable platform.

\section{System Architecture}

The proposed system has been designed in 3 levels: Design of Node Devices, Design of Base Station and Design of Controller. Figure 7 represent the algorithm of the full system. Abstraction of each level is discussed in following sections:

\section{Design of Node Devices}

Each room/component has a node device (Arduino uno/micro-controller or ESP8266 module (built-in microcontroller)) integrating with ESP8266 WiFi board mentioned above section. All the sensors and actuators are connected with microcontrollers. These microcontrollers act as slave devices, they execute the commands sent by central controllers or sent the sensed data by the sensors. The proposed system controls the switching devices (Different kinds of 
lights generally used in homes), cooling systems (AC, Fan), entertainment systems (TV, Sound System) and wash room devices (Water Heater, Shower). They all are connected either individually (ESP8266 module) or as bundle with microcontroller/ Arduino.

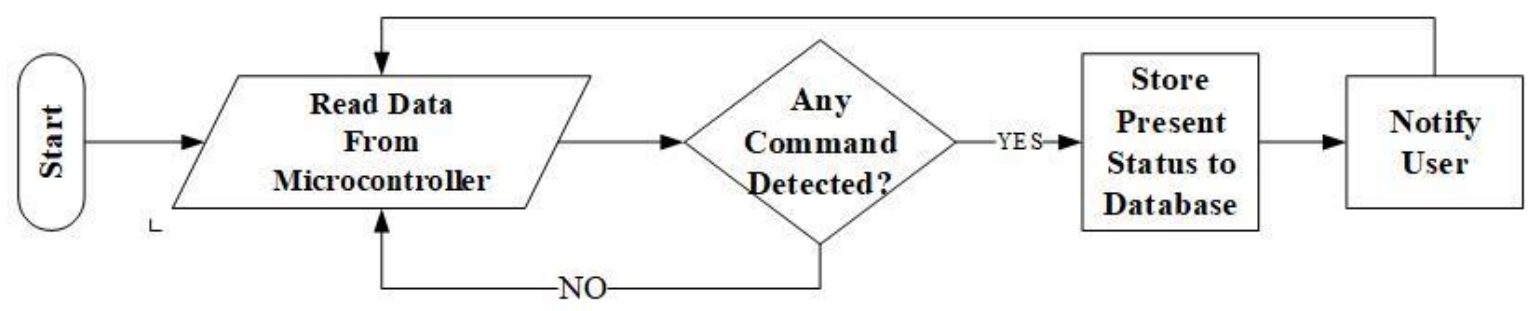

Figure 7. Algorithm of the proposed system

\section{Design of Base Station}

The core of control unit of the proposed system is Raspberry Pi. It is the base station (central controller) of the system and used as web server and application server. To make the system cost effective and user friendly, a Bluetooth module is also used for local users which is useful for internet failure in home network. Unlikely PC based or smart phone based home automation systems, with 40 i/o (I2C) pins called GPIO, HDMI and display ports it can be slave device and controller at the same time as well.

\section{Design of Proposed Controller}

For simplicity and keep the interface user friendly to potential users, both controller (web application and android application) use same interface shown in Figure 8. Figure 9 shows the user android interface. The first picture is the login page which enables the user to put username and password. After that the user will trigger the system to run the authorization. The next screenshot shows the home system interface which includes the buttons to display master bedroom, bedroom, garage and outdoor.

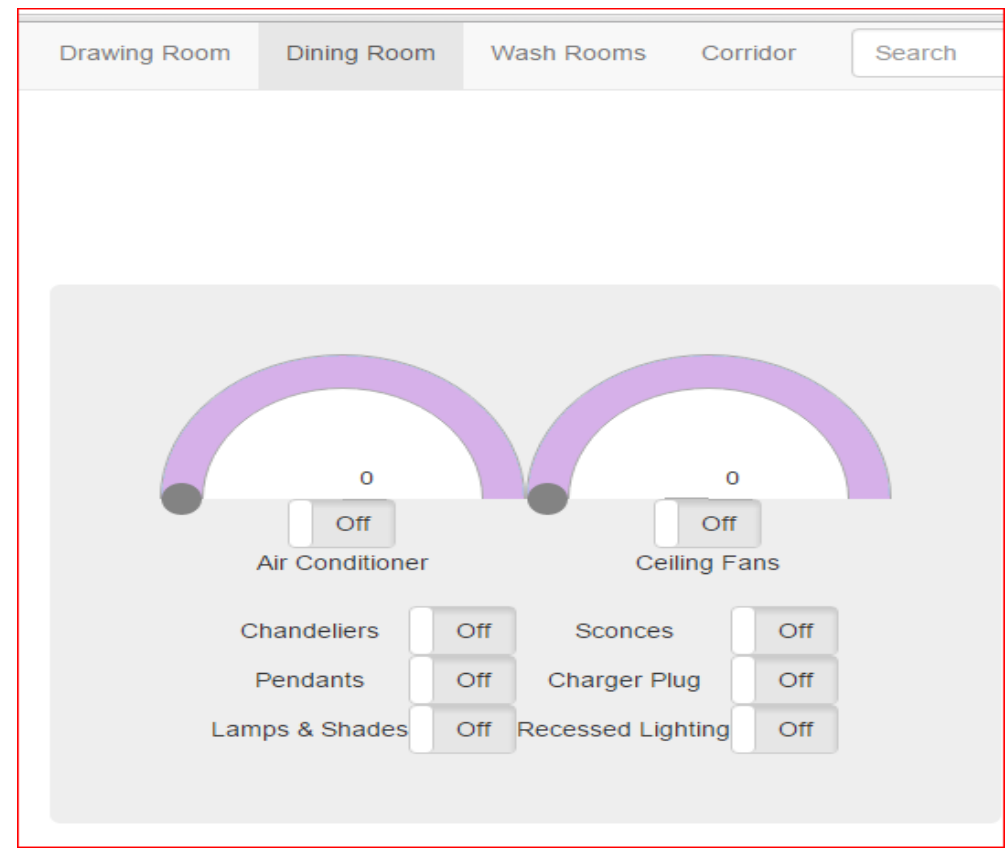

Figure 8. User interface of dining room and bedroom 


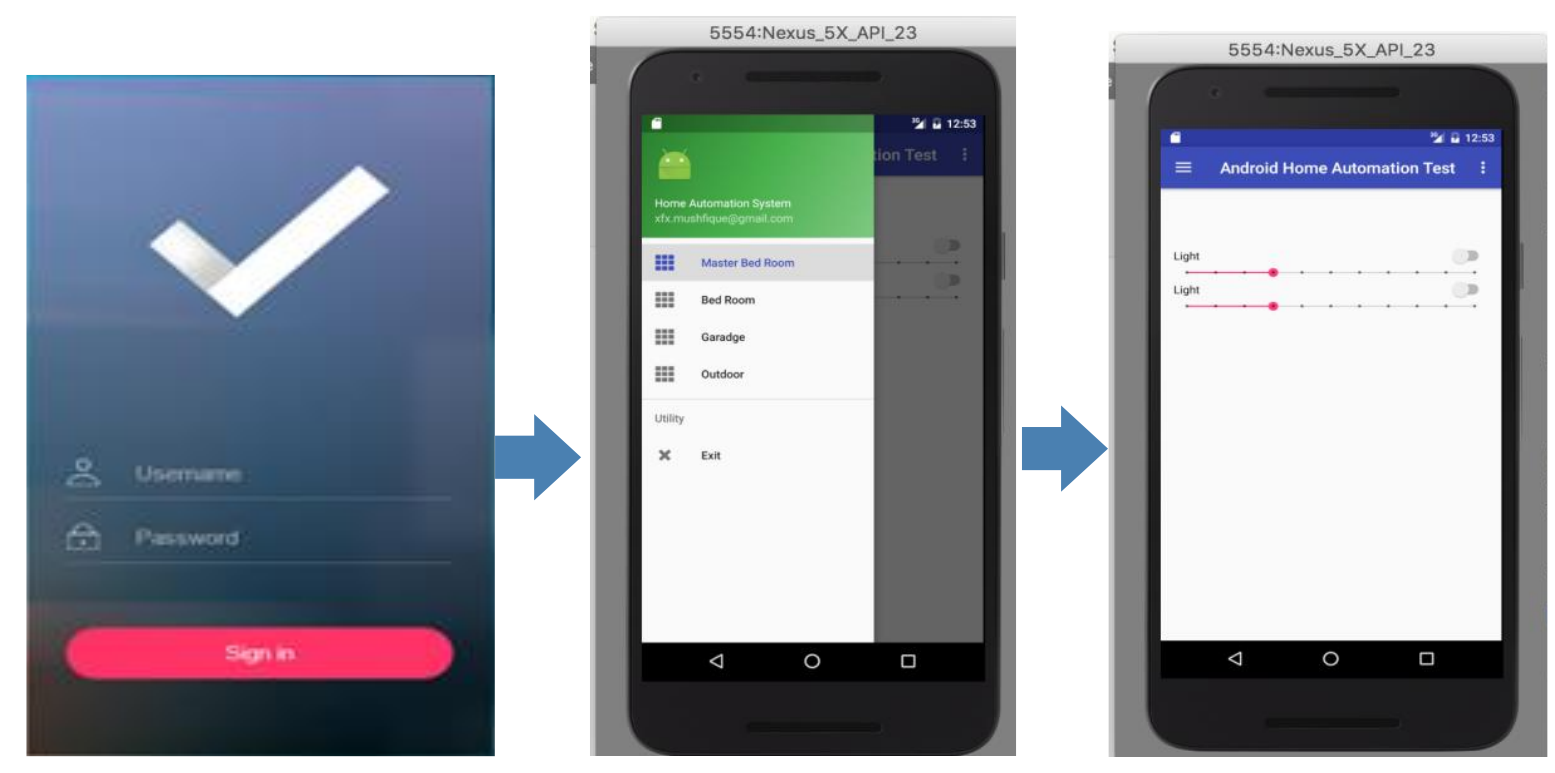

Figure 9. User interface of android application

The last screenshot of Figure 9 shows the intensity control of two lights. Additional bonus for mobile application can access the system through the Bluetooth of the mobile device. Both applications require user specific name and password to access the system generated by the home owner. Both ends use MySQL and PubNub API as database. Every time system stores its current data in MySQL DB (database). So even if the internet connection fails there will be no impact on state of the system. On reconnection, system will read data through MySQL DB, so user will get the most current state of the system. Figure 10 shows how a sample database entry looks like. The recent data are stored in these database entries. Like android device the system can be controlled from desktop also. Figure 11 shows the screenshot of user interface in pc software. First screenshot shows the login page and the last two pictures show the current status of light (on/off) and one can increase or decrease the intensity of light. The last interface of Figure 11 also shows the room temperature, humidity and air quality. The last interface screenshot also shows data for house.

\begin{tabular}{|rrllrl|}
\hline id & mode & name & description & value & type \\
\hline 1 & 1 & MASTER BED ROOM LAMP & Control room lamp & 60 & DIGITAL \\
\hline 2 & 0 & MASTER BED ROOM FAN & Control room fan & 0 & DIGITAL \\
\hline 3 & 1 & MASTER BED ROOM LED & Control room led & 100 & DIGITAL \\
\hline 4 & 1 & GARAGE DOOR & Control garage door & 100 & DIGITAL \\
\hline 5 & 0 & GARADGE LED & Control garage led & 0 & DIGITAL \\
\hline 6 & 1 & HALL ROOM LED & Control room led & 100 & DIGITAL \\
\hline 7 & 1 & KITCHEN LED & Control kitchen led & 100 & DIGITAL \\
\hline
\end{tabular}

Figure 10. Sample database entry 


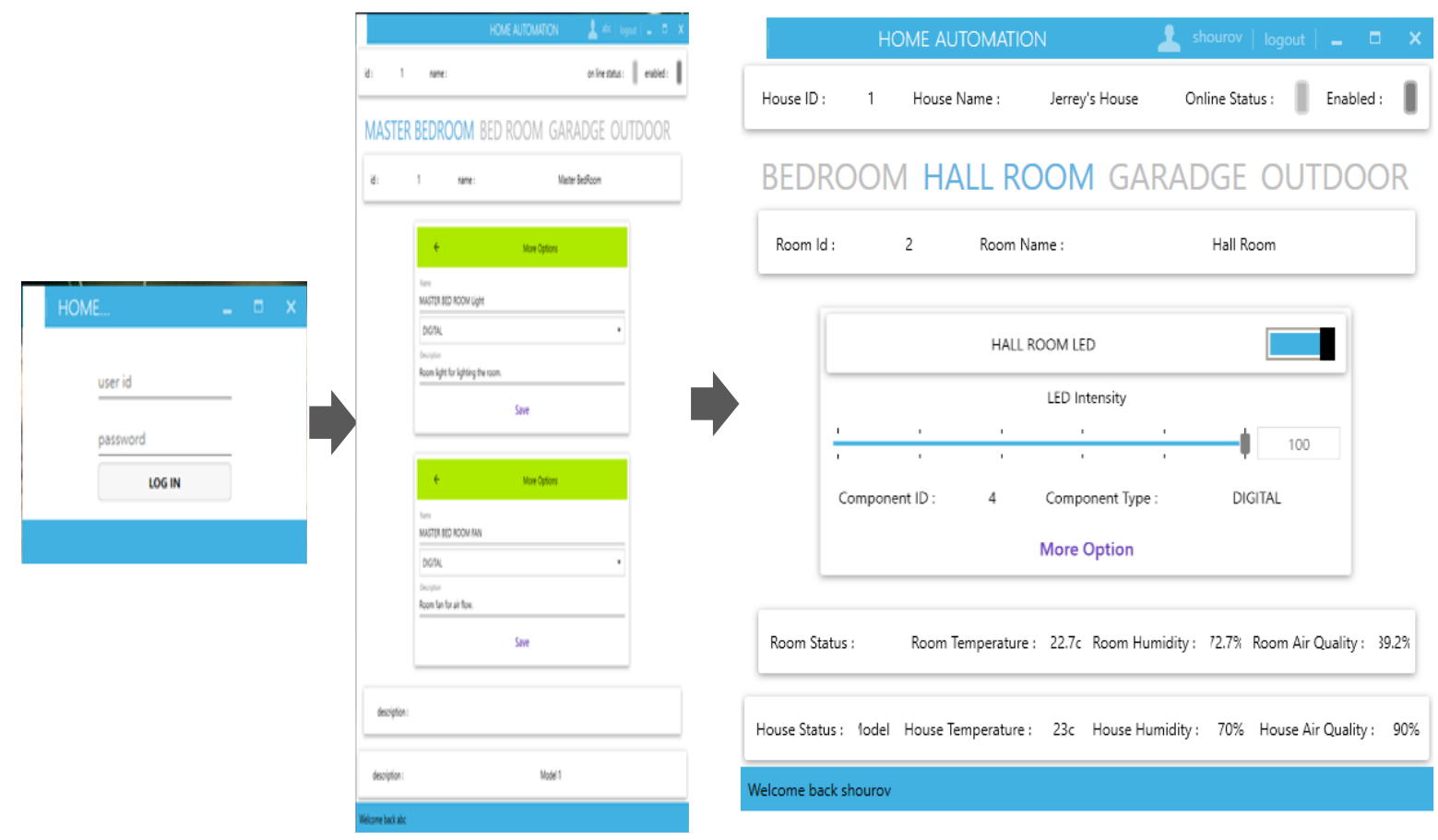

Figure 11. User interface in PC software

The sensor data can be downloaded from web interface which is shown in Figure 12. The humidity, temperature and air quality have been shown in the figure which were measured for the whole day. The sensor nodes were used to collect the measured data and after that the graph was plot in order to visualize the result properly. These data can be used for post processing to conduct the needs of different applications. With the help of the algorithms each sensor raw signal is used and Equation 1 is used to measure peak to peak values. After converting instantaneous values to rms values, average power is calculated using equation 2 [27].

$$
A_{x} V_{p p}=\frac{\left(\max _{v a l}-\min _{v a l}\right) * V_{\text {ref }}}{A D C_{v a l}}
$$

where $A_{x}$ is the analog port of the input signal, $V_{p p}$ is the peak to peak amplitude of the input signal, $\max _{\text {val }}$ is the maximum peak value of the signal, $\min _{v a l}$ is the minumum peak value of the signal, $V_{r e f}$ is the $5 \mathrm{~V}$ DC reference voltage of Arduino and $A D C_{\text {val }}$ is the 210 range of the ADC 1024.

$$
P_{a v g}=V_{r m s} \times I_{r m s} \times \cos \varphi
$$

After calculating power these values are shown in terms of graph in Figure 13. These data are measured for the whole day. Individual readings are there for TV, fridge, light, fan oven, AC (1 TON). The figure also shows the total power output. The data are measured for every single hour for one day. 


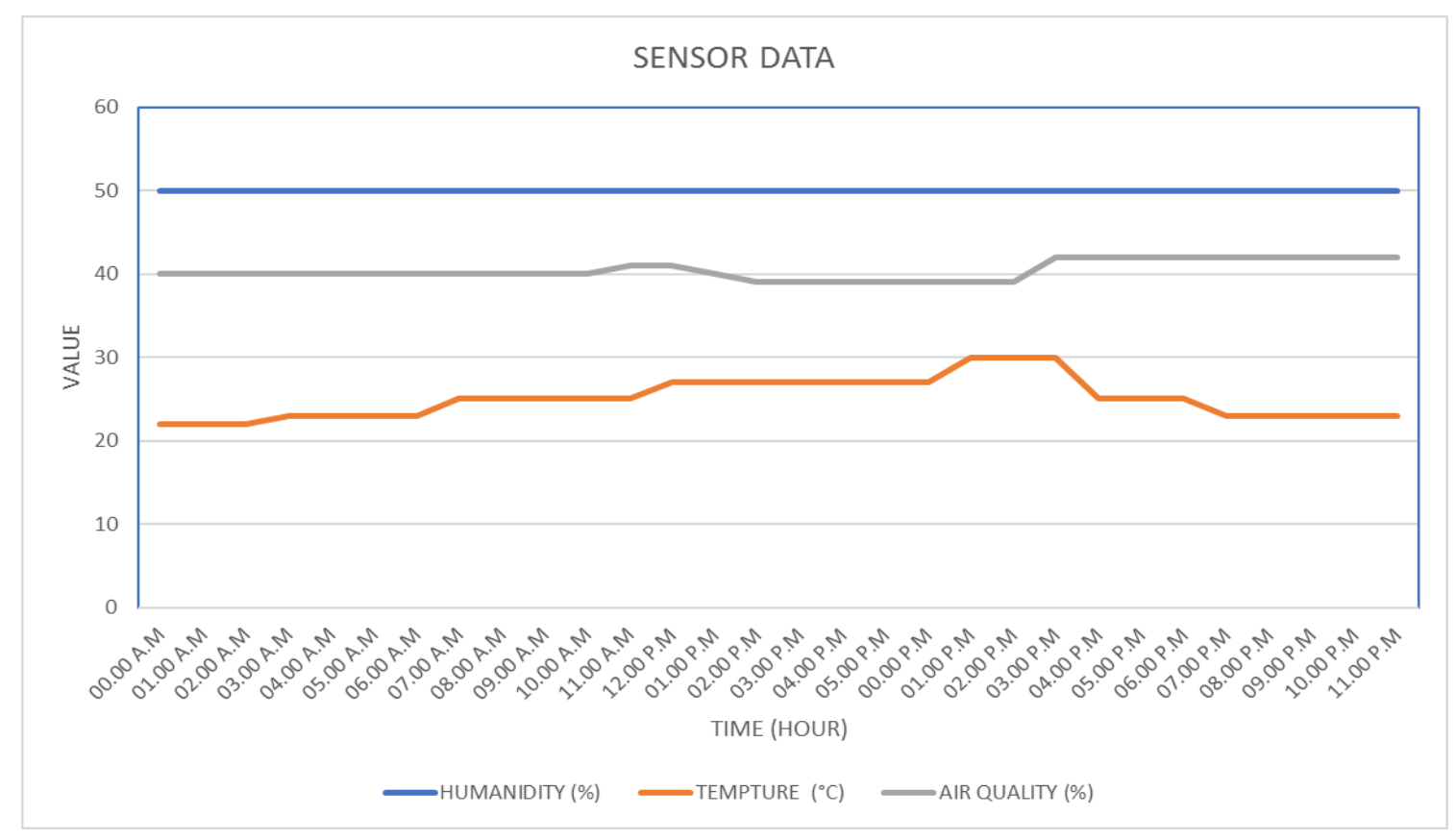

Figure 12. Sensors data

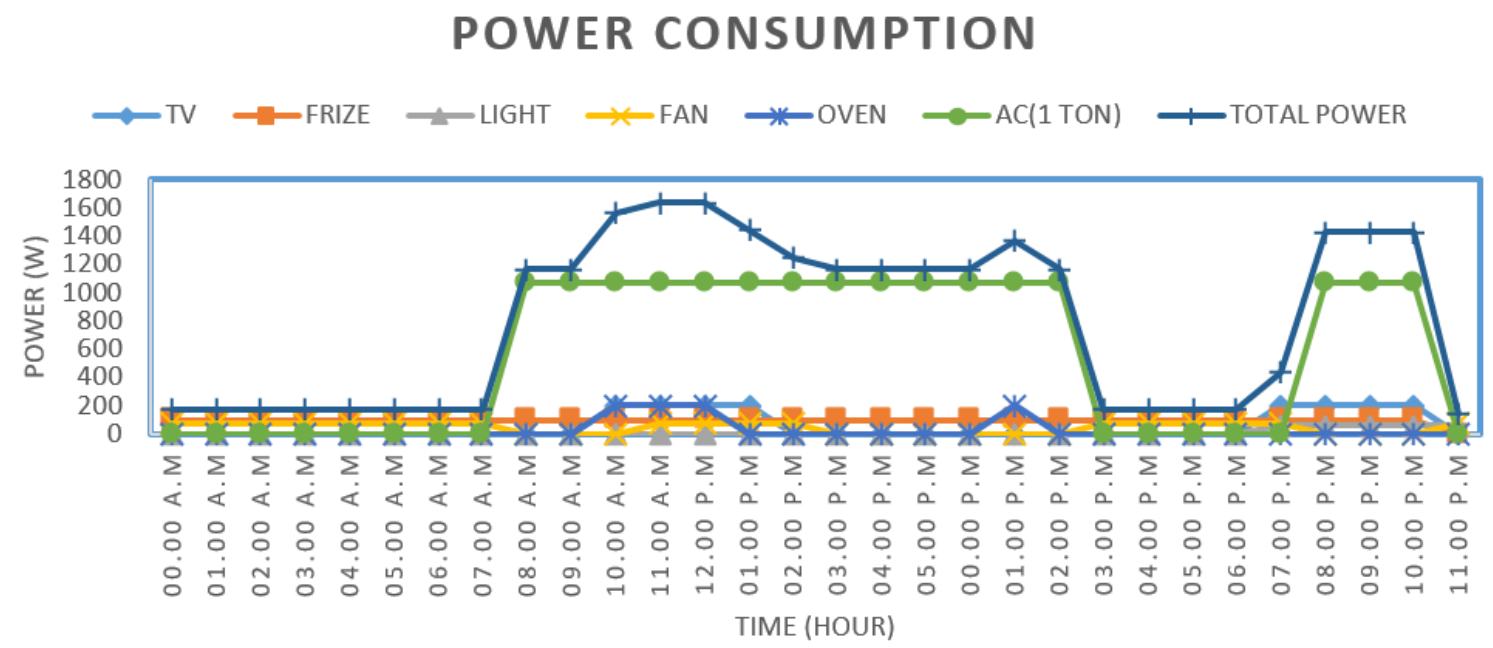

Figure 13. Power consumption of the appliances

Figure 14 and Figure 15 show the codes for connecting Wi-Fi module, which receives the commands from the android or web interface and execute by raspberry pi. The CT sensor (non-invasive current sensor) sends its data to the Arduino coupled with the Ethernet shield. The received data is processed by Arduino (Arduino IDE commands), and as a result, the energy consumed, and the price for the units consumed is sent to the database using the connected Ethernet shield. The load cell and the ADC module senses electricity bill and when the value reaches less than the set field, the bill amount is then send to the database for further utilization. Figure 16 shows the database for electricity bill. The information of energy consumption is then send by SMS to the user, which is shown in Figure 17. The overall control algorithm of this billing information is depicted by the flowchart of Figure 18. 


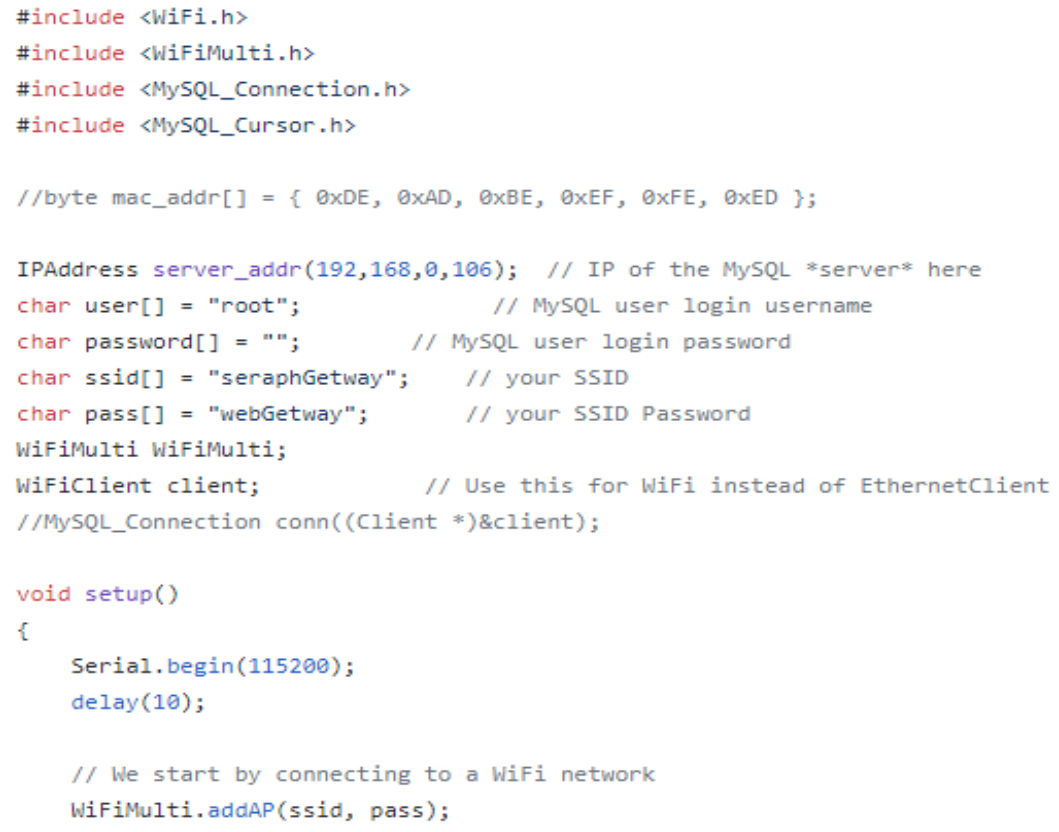

Figure 14. Code for connecting Wifi module (Part 1)

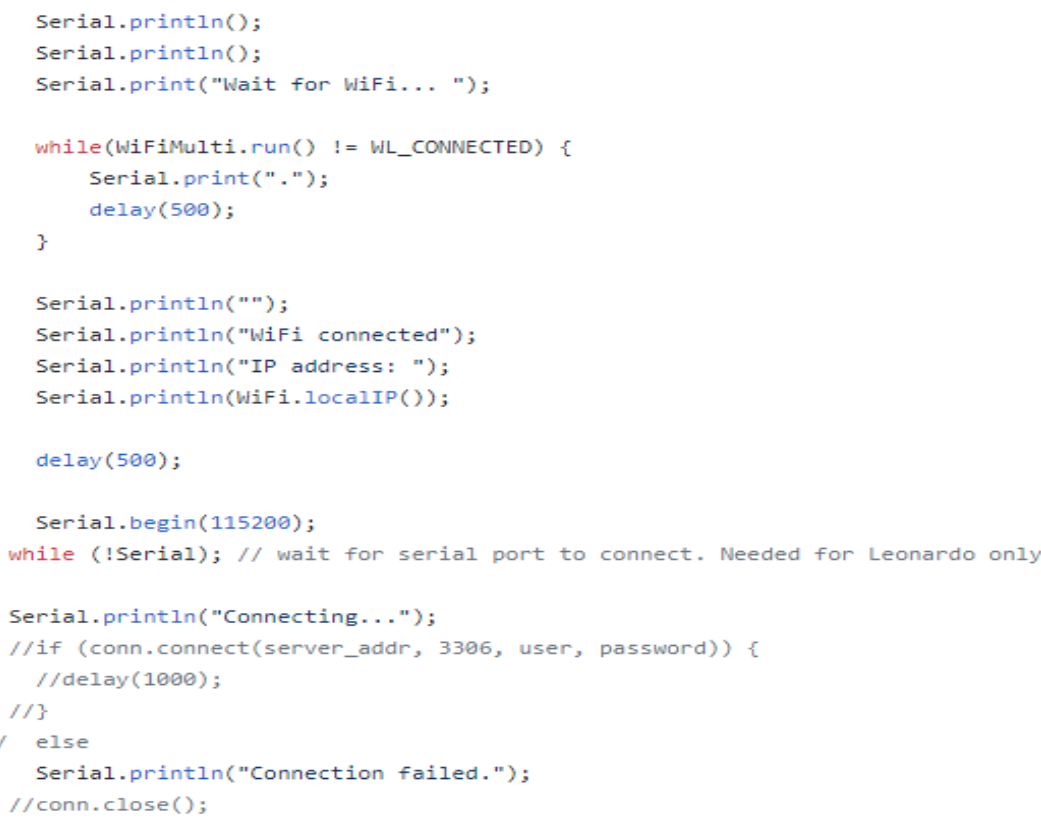

Figure 15. Code for connecting Wifi module (Part 2)

\begin{tabular}{|rrrr|}
\hline id & power & units_consumed & cost_agg \\
\hline 12 & 1832.7635 & 152.3200 & 1066.2400 \\
\hline 13 & 1567.2115 & 153.8200 & 1076.7400 \\
\hline 14 & 1461.3773 & 155.0800 & 1085.5600 \\
\hline 15 & 1517.1139 & 156.5900 & 1096.1300 \\
\hline 16 & 1908.7989 & 158.5100 & 1109.5100 \\
\hline
\end{tabular}

Figure 16. Database for the electricity bill consumed 


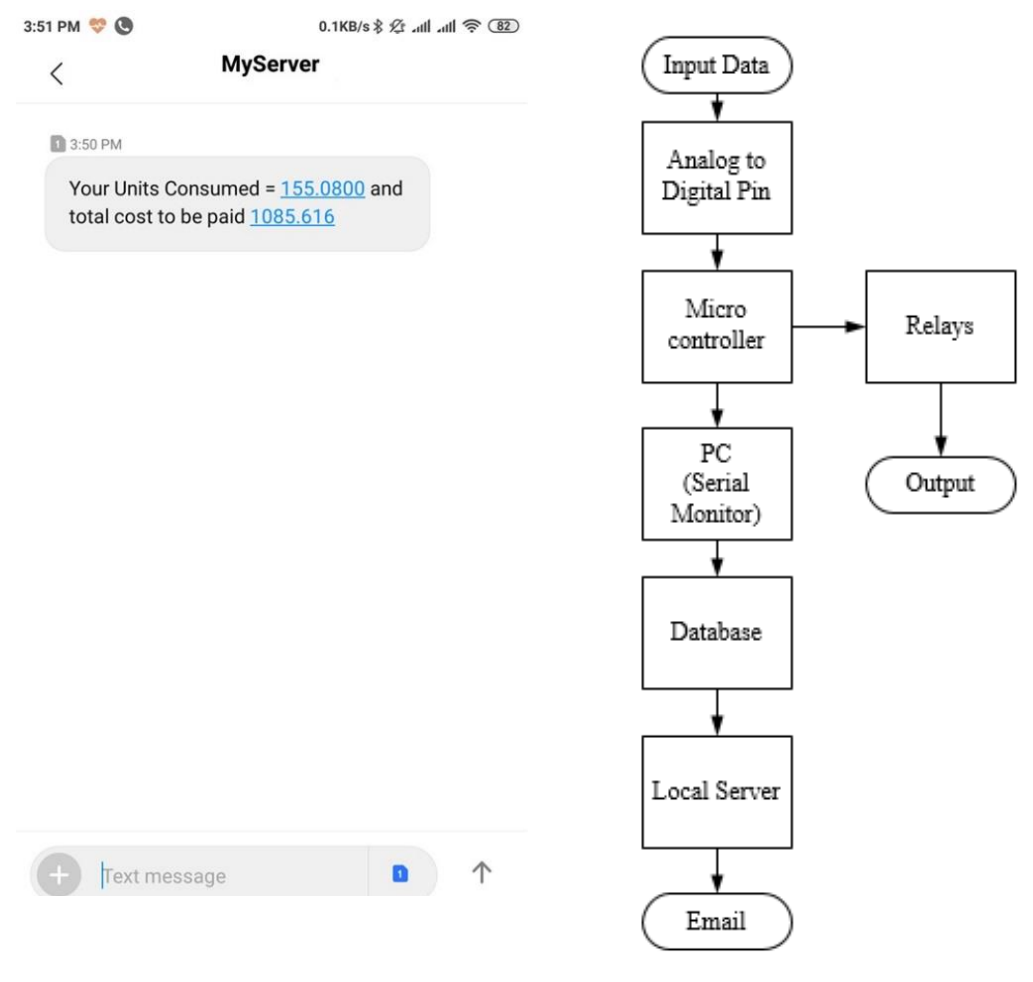

Figure 17. User notification messages about electricity bill
Figure 18. Flowchart for sending bill information to user

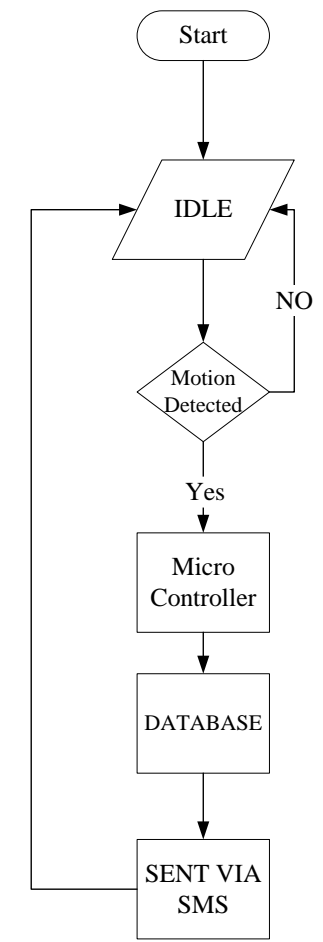

Figure 19. Flowchart of Home Security System

\section{Design of Home Security System}

Motion system is designed in such a way that it will be situated in the entrance of a house. These sensors will begin to work if any movement of humans are occurred in the range of the PIR sensor. A signal is sent to the micro-controller when motion sensor detects something. The owner will receive a voice call on his mobile phone and a SMS [24] about any intruder. To control the full house, other motion sensors can be placed and they will notify the controller about any intruder from those places.

\section{Design of Smart Guest Welcoming System}

This system is to design for welcoming guest at owner's house when he is not present. When any guest will arrive, a notification will be sent to the owner's mobile. Then the owner can turn on air-condition, light, fan etc. by pressing desired button.

The user will be notified also when any guest will leave his house. He can turn off the appliances with the help of micro-controller. Figure 19 shows the Home security and smart guest welcoming flowchart control.

\section{Design of Gas Leakage System}

When there is any gas detected by the gas detector sensor, the microcontroller will receive a signal. Then this signal will be stored on database for further instruction. After that the database will sent a predefined message to the user by using internet. Figure 20 shows the notification received by the user. In Figure 21 overall process of gas detection system is shown in a flowchart. 
In case of any fire, the flame sensor LM319 will sense and send a signal to the micro controller. After storing the information, a message is sent to database and to user by SMS. Figure 22 shows the notification of the fire which has been sent via SMS. Overall process has been shown by a flow chart in Figure 23.

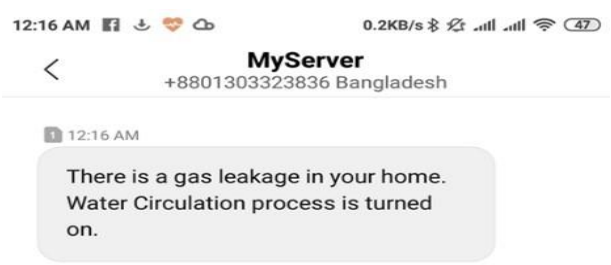

Text message D $~ \uparrow$

Figure 20. Notification of gas leakage via SMS from server

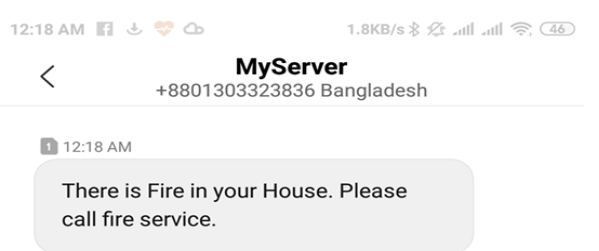

Text message

Figure 22. Notification of fire via SMS from server

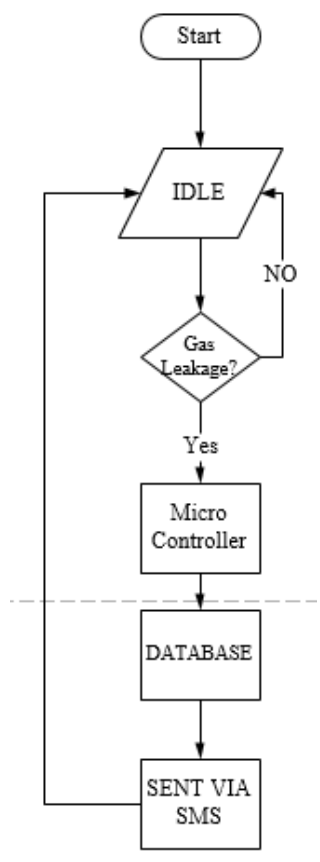

Figure 21. Flowchart of gas leakage detection

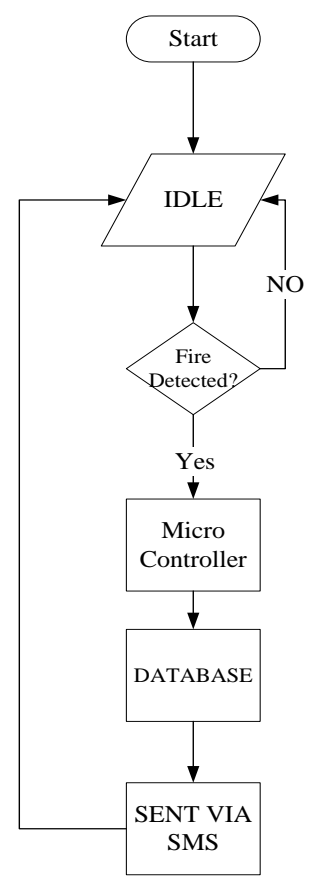

Figure 23. Flowchart of fire detection 


\section{Design of Rain Detection System}

The rain sensor detects any rain outside home and send a signal to the micro controller and the user by SMS. Figure 24 shows the SMS notification which was received by the user. Figure 25 shows the over flowchart of the above process.

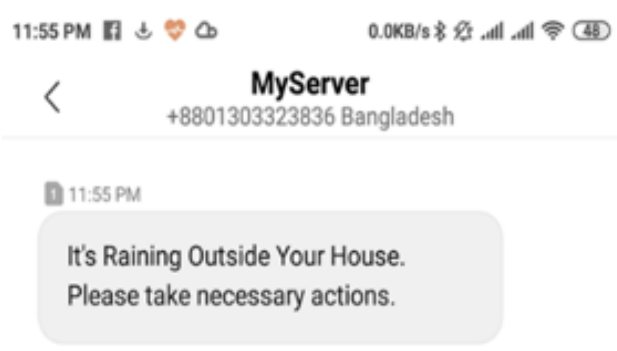

Figure 24. Notification of rain via SMS from server

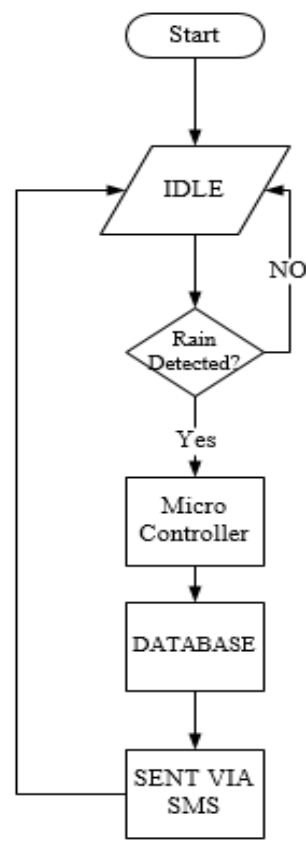

Figure 25. Flowchart of Rain

Detection System

\section{Comparison with Other Studies}

Table 1 shows the comparison of different previous studies with the proposed system. From the comparison, it is understood that the proposed system is better in all respects.

Table 1. Comparison of Previous Studies with Proposed System

\begin{tabular}{|l|c|c|c|c|c|}
\hline $\begin{array}{l}\text { Comparison } \\
\text { Indicators }\end{array}$ & $\begin{array}{c}\text { Reference } \\
{[\mathbf{1 2}]}\end{array}$ & $\begin{array}{c}\text { Reference } \\
{[\mathbf{1 3}]}\end{array}$ & $\begin{array}{c}\text { Reference } \\
{[\mathbf{1 4}]}\end{array}$ & $\begin{array}{c}\text { Reference } \\
{[\mathbf{1 5}]}\end{array}$ & $\begin{array}{c}\text { Proposed } \\
\text { System }\end{array}$ \\
\hline Cost & Medium & Very high & Medium & High & Low \\
\hline Control & No & Yes & Yes & Yes & yes \\
\hline Flexibility & Not flexible & $\begin{array}{c}\text { Not } \\
\text { flexible }\end{array}$ & Flexible & $\begin{array}{c}\text { Not } \\
\text { Flexible }\end{array}$ & $\begin{array}{c}\text { Very } \\
\text { Flexible }\end{array}$ \\
\hline Wireless Connection & Bluetooth & Wi-Fi & Bluetooth & Wi-Fi & $\begin{array}{c}\text { Wi-Fi and } \\
\text { Bluetooth }\end{array}$ \\
\hline $\begin{array}{l}\text { Energy } \\
\text { Consumption } \\
\text { monitoring }\end{array}$ & No & No & No & No & Yes \\
\hline
\end{tabular}




\section{Conclusions}

The proposed system with Arduino, Raspberry Pi, ESP8266 and a number of open-source software packages covers most of the present issues in home automation systems which ensures low cost, scalability, plug and play and configurable. The detailed design presented in this paper clearly demonstrates the potential of the proposed system. The system requires no knowledge on electrical systems or programming to install and manage the system as per requirement. As future work, the proposed system can be expanded in many aspects. For example, data visualization, management and analysis can be used to monitor the total usage power which will give the user more flexibility. Surveillance systems can also be included for security purpose and intruder detection.

\section{Acknowledgment}

The authors would like to acknowledge the financial support received from the Scientific Research Deanship, Taif University, KSA, through grant number 1-439-6072.

\section{References}

[1] A.C. Jose, and R. Malekian, "Improving smart home security: Integrating logical sensing into smart home," IEEE Sensors Journal, Vol. 17, No. 13, pp. 4269-4286, 2017.

[2] F. Moraes, A. Amory, N. Calazans, E. Bezerra, and J. Petrini, "Using the CAN protocol and reconfigurable computing technology for Web-based smart house automation," In: Symposium on Integrated Circuits and Systems Design, Pirenopolis, Brazil, pp. 38-43, 2017.

[3] R. Piyare, and M. Tazil, "Bluetooth based home automation system using cell phone," Paper presented at 15th International Symposium on Consumer Electronics (ISCE), IEEE, Singapore, 2011.

[4] V. Vujović, and M. Maksimović, "Raspberry Pi as a Sensor Web node for home automation," Computers \& Electrical Engineering, Vol. 44, pp. 153-171, 2015.

[5] I. Korkmaz, S. Metin, A. Gurek, C. Gur, C. Gurakin, and M. Akdeniz, "A cloud based and Android supported scalable home automation system," Computers \& Electrical Engineering, Vol. 43, pp. 112-128, 2015.

[6] "PubNub: Making Realtime Innovation Simple," PubNub, 2017 (n.d.). [Online]. Available: https://www.pubnub.com. [Accessed: October 2017].

[7] R. Ramya, C. Girisarathi, A. Jayaprakash, A. Karthikha and M. Srimalini "Implementation of smart home automation using raspberry PI," International Journal of Recent Trends in Engineering and Research, Vol. 3, pp. 122-125, 2018.

[8] "PrivateEyePi Project," Projects.privateeyepi.com, 2017 (n,d). [Online]. Available: http://www.projects.privateeyepi.com/home. [Accessed: May 2017].

[9] H. Lamine, and H. Abid, "Remote control of a domestic equipment from an Android application based on Raspberry PI card," Paper presented at 2014 15th International Conference on Sciences and Techniques of Automatic Control and Computer Engineering (STA), Hammamet, 2014.

[10] C. Andrews, "Easy as PI [Raspberry PI]," Engineering \& Technology, Vol. 8, No. 3, pp. 34-37, 2013.

[11] P. Martins, and J. McCann, "The Programmable City," Procedia Computer Science, Vol. 52, pp. 334-341, 2015.

[12] S. Ferdoush, and X. Li, "Wireless sensor network system design using Raspberry Pi and Arduino for environmental monitoring applications," Procedia Computer Science, Vol. 34, pp. 103-110, 2014. 
[13] S. Nasrin, and P.J. Radcliffe, "Novel protocol enables DIY home automation," In: 2014 Australasian Telecommunication Networks and Applications Conference (ATNAC), Southbank, Victoria, Australia, pp. 212-216, 2014.

[14] M.A.A. Milton, and A.A.S. Khan, "Web based remote exploration and control system using android mobile phone," Paper presented at 2012 International Conference on Informatics, Electronics \& Vision (ICIEV), Dhaka, Bangladesh, pp. 985-990, 2012.

[15] N. Sriskanthan, F. Tan, and A. Karande, "Bluetooth based home automation system," Microprocessors and Microsystems, Vol. 26, No. 6, pp. 281-289, 2002.

[16] A.R. Al-Ali, and M. Al-Rousan, "Java-based home automation system," IEEE Transactions on Consumer Electronics, Vol. 50, No. 2, pp. 498-504, 2004.

[17] N. Vikram, K.S. Harish, M.S. Nihaal, R. Umesh, A. Shetty, and A. Kumar, "A low cost home automation system using wi-fi based wireless sensor network incorporating internet of things IOT)," In: 2017 IEEE 7th International Advance Computing Conference (IACC), Hyderabad, India, pp. 174-178, 2017.

[18] H. Singh, V. Pallagani, V. Khandelwal, and U. Venkanna, "IoT based smart home automation system using sensor node," Paper presented at 2018 4th International Conference on Recent Advances in Information Technology (RAIT), Dhanbad, India, pp. 1-5, 2018.

[19] P. Kumar, and U.C. Pati, "IoT based monitoring and control of appliances for smart home," Paper presented at 2016 IEEE International Conference on Recent Trends in Electronics, Information \& Communication Technology (RTEICT), Bangalore, pp. 1145-1150, 2016.

[20] F.M. Albatsh, S. Mekhilef, S. Ahmad, and H. Mokhlis, "Fuzzy logic based UPFC and laboratory prototype validation for dynamic power flow control in transmission lines," IEEE Transactions on Industrial Electronics, Vol. 64, No. 12, pp. 9538-9548, 2017.

[21] F.M. Albatsh, S. Ahmad, S. Mekhilef, H. Mokhlis, and M. Hassan, "Optimal placement of unified power flow controllers to improve dynamic voltage stability using power system variable based voltage stability indices," PLOS One, Vol. 10, No. 4, pp. 14521468, 2015.

[22] S. Ahmad, F.M. Albatsh, S. Mekhilef, and H. Mokhlis, "Fuzzy based controller for dynamic Unified Power Flow Controller to enhance power transfer capability," Energy Conversion and Management, Vol. 79, pp. 652- 665, 2014.

[23] F.M. Albatsh, S. Ahmad, S. Mekhilef, I. Alhamrouni, and M.F.A. Hamid. "Power flow control using fuzzy based UPFC under different operating conditions," Journal of Electrical Systems, Vol. 13, No. 2, pp. 398-414, 2017.

[24] F.M.G.K.D. Sukmana, H. Teja, "Wireless and mobile (APWIMOB)," Paper Presented at 2015 IEEE Asia Pacific Conference, pp. 183-187, 2015.

[25] R. Kodali, V. Jain, S. Bose and L. Boppana, "IoT based smart security and home automation system," Paper presented at 2016 International Conference on Computing, Communication and Automation (ICCCA), 2016.

[26] N. Vikram, K. Harish, M. Nihaal, R. Umesh, A. Shetty, and A. Kumar, "A low cost home automation system using Wi-Fi based wireless sensor network incorporating internet of things (IoT)," Paper presented at 2017 IEEE 7th International Advance Computing Conference (IACC), 2017.

[27] R. Bharathi, M.E. Madhushree, and P. Kumari, "Power Consumption Monitoring System using IOT," International Journal of Computer Applications, Vol. 173, No. 5, pp. 23-25, 2017. 